\title{
Analysis of copy number variations at 15 schizophrenia-associated loci
}

Elliott Rees, James T. R. Walters, Lyudmila Georgieva, Anthony R. Isles, Kimberly D. Chambert, Alexander L. Richards, Gerwyn Mahoney-Davies, Sophie E. Legge, Jennifer L. Moran, Steven A. McCarroll, Michael C. O'Donovan, Michael J. Owen and George Kirov

\section{Background}

A number of copy number variants (CNVs) have been suggested as susceptibility factors for schizophrenia. For some of these the data remain equivocal, and the frequency in individuals with schizophrenia is uncertain.

\section{Aims}

To determine the contribution of CNVs at 15 schizophreniaassociated loci (a) using a large new data-set of patients with schizophrenia $(n=6882)$ and controls $(n=6316)$, and (b) combining our results with those from previous studies.

\section{Method}

We used Illumina microarrays to analyse our data. Analyses were restricted to 520766 probes common to all arrays used in the different data-sets.

\section{Results}

We found higher rates in participants with schizophrenia than in controls for 13 of the 15 previously implicated CNVs.
Six were nominally significantly associated $(P<0.05)$ in this new data-set: deletions at 1q21.1, NRXN1, 15q11.2 and 22q11.2 and duplications at 16p11.2 and the Angelman/ Prader-Willi Syndrome (AS/PWS) region. All eight AS/PWS duplications in patients were of maternal origin. When combined with published data, 11 of the 15 loci showed highly significant evidence for association with schizophrenia $\left(P<4.1 \times 10^{-4}\right)$

\section{Conclusions}

We strengthen the support for the majority of the previously implicated CNVs in schizophrenia. About $2.5 \%$ of patients with schizophrenia and $0.9 \%$ of controls carry a large, detectable CNV at one of these loci. Routine CNV screening may be clinically appropriate given the high rate of known deleterious mutations in the disorder and the comorbidity associated with these heritable mutations.

\section{Declaration of interest}

None.
Copy number variants (CNVs) are chromosomal rearrangements involving large segments of DNA (from 1000 and up to several million base pairs in length) that can be deleted, duplicated, inverted or translocated. A number of pathogenic CNVs are known to cause clinically recognisable syndromes, such as Williams-Beuren syndrome (WBS), Angelman/Prader-Willi syndrome (AS/PWS) and velocardiofacial syndrome (VCFS). ${ }^{1}$ Some CNVs are associated with a highly variable phenotype, which often includes both developmental and neuropsychiatric disorders. ${ }^{1}$ For example, 22q11.2 deletions, the first CNV to be associated with schizophrenia, ${ }^{2}$ also causes VCFS, which is characterised by intellectual disability and a number of medical problems such as palatal and skeletal anomalies and cardiac defects. ${ }^{3,4}$ Several large and rare CNVs have now been implicated in the aetiology of schizophrenia, ${ }^{5-13}$ reviewed by Malhotra \& Sebat. ${ }^{14}$ They have been shown to substantially increase risk for the development of the disorder, from twofold to over 60 -fold. ${ }^{14}$ Most of them have been shown to also increase risk for other disorders, such as autism spectrum disorders, intellectual deficit, developmental delay and epilepsy. ${ }^{1,14,15}$

As some of the CNVs are very rare (found in less than 1 in 1000 patients), notwithstanding the relatively large data-sets examined so far (ranging between 5000 and 14000 people with schizophrenia), it is not clear that all those that have been implicated are true risk factors for the disorder. Thus, for nine of the loci that have received the strongest support in the literature ${ }^{14}$ fewer than 15 observations have been made in people with schizophrenia. These nine loci are: duplications at 1q21.1, ${ }^{10}$ the WBS region, ${ }^{16}$ the AS/PWS region, ${ }^{6}$ at VIPR $2,{ }^{10,17}$ at $16 \mathrm{p} 13.11^{7}$ and deletions at $3 \mathrm{q} 29,{ }^{10,12}$ distal $16 \mathrm{p} 11.2,{ }^{18} 17 \mathrm{q} 12^{19}$ and $17 \mathrm{p} 12 .^{20}$ The evidence for six loci is based only on single, albeit very large, studies: deletions at $17 \mathrm{q} 12,{ }^{19}$ distal $16 \mathrm{p} 11.2^{18}$ and $17 \mathrm{p} 12^{20}$ and duplications at $1 \mathrm{q} 21.1,{ }^{10} \mathrm{AS} / \mathrm{PWS}$ region ${ }^{6}$ and at $16 \mathrm{p} 13.11 .^{7}$ Moreover, the role of two loci, deletions at $15 \mathrm{q} 11.2$ and duplications at $16 \mathrm{p} 13.11$, has recently been challenged by a study that indicated that the rates among controls might be higher than originally reported, ${ }^{5}$ and no excess in individuals with schizophrenia was seen for these two CNVs in one of the largest studies. ${ }^{10}$ Finally, each previous report focused on the identification of one, or only a small number of CNV loci, and several reports used partially overlapping data-sets. Consequently, the rate of all previously reported risk CNVs has not yet been evaluated in a single independent data-set that is not biased by the inclusion of the original discovery sample. We set out to evaluate these specific CNVs in a sample of 6882 people with schizophrenia that passed quality control. This represents the largest single CNV data-set yet reported in schizophrenia, and nearly doubles the sample size of patients from the total world literature for many loci. For control individuals, we used publicly available data from 6316 samples genotyped on arrays with a resolution similar to the case group. These data-sets are completely independent from samples used in previous studies or reviews that established the rates of these CNVs in schizophrenia.

\section{Method}

\section{Samples}

Case group

We collected data on patients with schizophrenia (case group) in two waves, which we call (a) CLOZUK and (b) CardiffCOGS 
(Cardiff Cognition in Schizophrenia). The CLOZUK sample $(n=6558)$ consists of individuals taking the antipsychotic clozapine. In the UK, clozapine is reserved for patients with treatment-resistant schizophrenia, who provide regular blood samples to allow detection of adverse drug effects. Through collaboration with Novartis, the manufacturer of a proprietary form of clozapine, we acquired anonymised blood samples from people on clozapine. Participants (71\% male) were aged $18-90$, with a recorded diagnosis of treatment-resistant schizophrenia according to the clozapine registration forms completed by their psychiatrists. The use of these anonymised samples for genetic association studies was approved by the local ethics committee. The CLOZUK sample has been described elsewhere. ${ }^{21}$ The CardiffCOGS $(n=571)$ is a sample of patients with clinically diagnosed schizophrenia recruited from community, in-patient and voluntary sector mental health services in the UK. Interview with the Schedules for Clinical Assessment in Neuropsychiatry (SCAN) instrument ${ }^{22}$ and case-note review was used to arrive at a best-estimate lifetime diagnosis according to DSM-IV criteria. ${ }^{23}$

\section{Control group}

As controls we used publicly available data downloaded from dbGAP (www.ncbi.nlm.nih.gov/gap). To avoid CNV detection biases between arrays with different probe densities and platforms, we chose data-sets genotyped with Illumina arrays that had a high overlap with the probes used to genotype the case group. The following data-sets were used: 1491 participants from the USA that took part in a study on smoking cessation, 3102 participants from the USA who took part in a study on melanoma and 1869 participants from Germany who took part in a study of refractive error (KORA study). Participants in the 'smoking' and 'melanoma' data-sets are cases or controls from these studies, whereas the KORA data-set is a population-based study where participants had refractive error measurements. The ethnicities of participants were derived from principal components analysis (PCA). A total of $91.4 \%$ of samples that passed quality control were from individuals of European ancestry. Full details on these data-sets are presented in the online supplement (section 1).

\section{Genotyping and quality control filtering}

Raw intensity files from each data-set were independently processed to account for potential batch effects. PennCNV ${ }^{24}$ was used for CNV detection. To avoid cross-platform biases, we restricted CNV calling to the 520766 probes present on all arrays used. Samples were excluded if any one of the following standard quality control statistics constituted an outlier within their source data-set: $\log \mathrm{R}$ ratio (LRR) standard deviation, B-allele frequency (BAF) drift, wave factor (WF) and total number of CNVs (online supplement, section 2). Out of 13591 samples with array data, 393 were excluded due to poor quality control or for being duplicates of the same individual after testing for identity-by-descent. The $8.6 \%$ non-European participants were retained in the analysis, to ensure that our data were comparable with those in recent reviews of $\mathrm{CNV}$ loci in schizophrenia. ${ }^{14}$ The numbers and ethnicities of these participants are listed in online Table DS2. The final numbers after exclusions for quality control and duplicates was 6882 in the case group and 6316 in the control group. The quality control process for individual CNVs is detailed in the online supplement (section 2). Briefly, CNVs were included if they were $>10 \mathrm{~kb}$ in size and had a frequency $<1 \%$, (applying filters with PLINK version 1.07). ${ }^{25}$ All CNVs were subsequently required to pass a median $z$-score outlier method of validation ${ }^{26}$ that helps to remove false-positive CNVs, and to identify any missed CNVs.

\section{Statistical analysis}

For the analysis of CNV loci we used Fisher's exact test (1-tailed as we were testing prior hypotheses). We performed a meta-analysis by adding our new data to those in the literature, applying a 2-tailed Fisher's exact test to the combined data. As these CNVs have been shown to be subjected to strong negative selection pressure, their frequencies in the population essentially reflect the mutation rate and selection pressure operating against them. ${ }^{1,27}$ They are therefore less likely to be subjected to population stratification caused by genetic drift, in the way common variants are (although there are some examples of ethnic differences in the mutation rates). Therefore, in the interests of clarity, we do not stratify the sample by ethnicity but provide the full breakdown of the data in the different ethnic groups in online Table DS3. The results do not change appreciably if they are restricted to only the European populations that comprise $91.4 \%$ of the sample.

There is no accepted convention as to what constitutes genome-wide significance for a CNV locus. Girirajan and colleagues $^{1}$ reported 72 recurrent $\mathrm{CNV}$ s that can cause a neurodevelopmental disorder, although in total, 120 genomic regions are potentially prone to recurrent CNVs because they are flanked by segments of high homology, called segmental duplications. ${ }^{28}$ This suggests that a Bonferroni correction for multiple testing of recurrent $\mathrm{CNVs}$ (those flanked by segmental duplications) might require a $P$-value of $<4.1 \times 10^{-4}$ to be accepted as a significant association for this type of $\mathrm{CNV}(P=0.05 / 120)$. Regarding associations with individual genes, a conservative Bonferroni correction would require correcting for the testing of $\sim 20000$ genes, or $P<2.5 \times 10^{-6}(P=0.05 / 20000)$.

\section{Choice of CNVs for analysis}

The list of previously implicated CNVs was taken from the largest meta-analysis to date. ${ }^{14}$ To this list we added three loci: exondisrupting deletions at the NRXN1 gene, as there is consensus that they increase risk for developing schizophrenia; ${ }^{9,10,13}$ deletions at distal 16p11.2, the evidence for which was published after the above review; ${ }^{18}$ and duplications at the WBS region, a locus that just failed to reach significance in that review, but received support in a subsequent study. ${ }^{16}$ For duplications at the AS/PWS region, we tested their parental origin using a DNA methylation-sensitive high-resolution melting curve analysis, ${ }^{29}$ as previous research suggested that the maternal ones are specifically implicated ${ }^{6}$ (online supplement, section 5).

\section{Results}

The rates of CNVs among the case and control groups are presented in Table 1. For 13 out of the 15 CNVs, we found higher rates in the case than in the control group. For six of these, the difference was nominally significant in this new sample alone (Table 1).

Of the loci where previous evidence was modest, the most striking result was for AS/PWS duplications, where we found eight in patients and none in controls $(P=0.0055)$. When combined with previous data (Table 2, for a more detailed version that includes results from previous studies see online Table DS6), this $\mathrm{CNV}$ is now a strongly supported schizophrenia risk variant. Moreover, as this is an imprinted locus, we tested the parental origin of our eight duplications, and all were shown to be maternal in origin, similar to the original publication. ${ }^{6}$ Duplication at $16 \mathrm{p} 13.11$, another previously weakly associated CNV, now also shows strong evidence in the combined data (Table 2). 


\begin{tabular}{|c|c|c|c|c|c|c|c|}
\hline \multirow[b]{2}{*}{ Locus } & \multirow[b]{2}{*}{ Position in $\mathrm{Mb}$} & \multicolumn{2}{|c|}{ Case group $(n=6882$ ) } & \multicolumn{2}{|c|}{ Control group $(n=6316)$} & \multirow[b]{2}{*}{ OR $(95 \% \mathrm{Cl})$} & \multirow[b]{2}{*}{$P$} \\
\hline & & CNVs, $n$ & Frequency, \% & CNVs, $n$ & Frequency, \% & & \\
\hline 1q21.1 del & chr1:146,57-147,39 & 12 & 0.17 & 1 & 0.016 & $11.03(1.43-84.86)$ & 0.0027 \\
\hline 1q21.1 dup & chr1:146,57-147,39 & 8 & 0.12 & 5 & 0.079 & $1.47(0.48-4.49)$ & 0.35 \\
\hline NRXN1 del & chr2:50,15-51,26 & 11 & 0.16 & 0 & 0.00 & NA $(1.25-\infty)$ & $7.7 \times 10^{-4}$ \\
\hline $3 q 29$ del & chr3:195,73-197,34 & 4 & 0.058 & 0 & 0.00 & NA $(0.44-\infty)$ & 0.074 \\
\hline WBS dup & chr7:72,74-74,14 & 3 & 0.044 & 1 & 0.016 & $2.75(0.29-26.48)$ & 0.35 \\
\hline VIPR2 dup & chr7:158,82-158,94 & 1 & 0.015 & 6 & 0.095 & $0.15(0.02-1.27)$ & 0.99 \\
\hline $15 q 11.2$ del & chr15:22,80-23,09 & 44 & 0.64 & 26 & 0.41 & $1.56(0.96-2.53)$ & 0.046 \\
\hline AS/PWS dup & chr15:24,82-28,43 & 8 & 0.12 & 0 & 0.00 & NA $(0.90-\infty)$ & 0.0055 \\
\hline $15 q 13.3 \mathrm{del}$ & chr15:31,13-32,48 & 4 & 0.058 & 2 & 0.032 & $1.84(0.34-10.03)$ & 0.38 \\
\hline 16p13.11 dup & chr16:15,51-16,30 & 24 & 0.35 & 12 & 0.19 & $1.84(0.92-3.68)$ & 0.056 \\
\hline 16p11.2 distal del & chr16:28,82-29,05 & 0 & 0.00 & 2 & 0.032 & NA (0-3.82) & 1 \\
\hline 16p11.2 dup & chr16:29,64-30,20 & 27 & 0.39 & 0 & 0.00 & NA $(3.09-\infty)$ & $2.3 \times 10^{-8}$ \\
\hline 17p12 del & chr17:14,16-15,43 & 4 & 0.058 & 3 & 0.047 & $1.22(0.27-5.47)$ & 0.55 \\
\hline $17 q 12$ del & chr17:34,81-36,20 & 1 & 0.015 & 0 & 0.00 & NA $(0.11-\infty)$ & 0.52 \\
\hline $22 q 11.2$ del & chr22:19,02-20,26 & 20 & 0.29 & 0 & 0.00 & NA $(2.28-\infty)$ & $2.2 \times 10^{-6}$ \\
\hline Totals & & 171 & 2.48 & 58 & 0.92 & & $1.4 \times 10^{-12}$ \\
\hline
\end{tabular}

The only instances in our study where CNVs were more common in the control than in the case group concern duplications at VIPR2 and deletions at distal 16p11.2. In neither case is the excess of CNVs in the control group significant. In the case of VIPR2, meta-analysis is no longer supportive, whereas the evidence for association at distal 16p11.2 remains nominally significant (Table 2 and Table DS6). Analysis of only individuals of European descent gave essentially the same results. The distribution of CNVs in the different data-sets and ethnic groups is presented in Table DS3.

In the present sample, which is not subject to the potential bias of including the original studies that discovered the associations, about $2.5 \%$ of the case group and $0.9 \%$ of the control group carry one of the CNVs in Table 1, a highly significant excess $\left(P=1.4 \times 10^{-12}\right)$. Only four individuals in the case group carry two of these CNVs (online supplement, section 6).
In the analysis of all 15 loci in the combined data (Table 2), all but one of the CNVs showed significant evidence of association. For 11 of these, the significance surpasses the threshold for multiple testing correction that we suggest in the Method $\left(P<4.1 \times 10^{-4}\right)$. For many of them the statistical significance is greatly improved compared with the previous results, most strikingly for 15q11.2, AS/PWS, 16p13.11, 16p11.2 and 22q11.2, where the $P$-values were strengthened by several orders of magnitude.

\section{Discussion}

In an analysis of the largest single schizophrenia sample to date, we establish more accurate estimates of risk from individual CNVs in an independent sample, and estimate the total burden of susceptibility conferred by this group of CNVs. The vast majority

\begin{tabular}{|c|c|c|c|c|c|}
\hline \multirow[b]{2}{*}{ Locus } & \multirow[b]{2}{*}{$P$-value in previous studies } & \multicolumn{2}{|c|}{ CNV frequency, \% $(n / N)$} & \multirow[b]{2}{*}{ OR $(95 \% \mathrm{Cl})$} & \multirow[b]{2}{*}{$P$} \\
\hline & & Case group & Control group & & \\
\hline 1q21.1 del & $1.3 \times 10^{-9}$ & $0.17(33 / 19056)$ & $0.021(17 / 81821)$ & 8.35 (4.65-14.99) & $4.1 \times 10^{-13}$ \\
\hline 1q21.1 dup & $2.0 \times 10^{-4}$ & $0.13(21 / 16247)$ & $0.037(24 / 64046)$ & $3.45(1.92-6.20)$ & $9.9 \times 10^{-5}$ \\
\hline NRXN del & $7.9 \times 10^{-9}$ & $0.18(33 / 18762)$ & $0.020(10 / 51$ 161) & 9.01 (4.44-18.29) & $1.3 \times 10^{-11}$ \\
\hline $3 q 29$ del & $2.3 \times 10^{-8}$ & 0.082 (14/17 005) & 0.0014 (1/69 965) & 57.65 (7.58-438.44) & $1.5 \times 10^{-9}$ \\
\hline WBS dup & $5.5 \times 10^{-5}$ & $0.066(14 / 21269)$ & $0.0058(2 / 34455)$ & 11.35 (2.58-49.93) & $6.9 \times 10^{-5}$ \\
\hline VIPR2 dup & 0.006 & $0.11(15 / 14218)$ & $0.069(17 / 24815)$ & $1.54(0.77-3.09)$ & 0.27 \\
\hline $15 q 11.2$ del & $2.2 \times 10^{-7}$ & $0.59(116 / 19547)$ & $0.28(227 / 81802)$ & $2.15(1.71-2.68)$ & $2.5 \times 10^{-10}$ \\
\hline AS/PWS dup & 0.014 & $0.083(12 / 14464)$ & $0.0063(3 / 47686)$ & $13.20(3.72-46.77)$ & $5.6 \times 10^{-6}$ \\
\hline $15 q 13.3 \mathrm{del}$ & $2.1 \times 10^{-11}$ & $0.14(26 / 18571)$ & 0.019 (15/80 422) & $7.52(3.98-14.19)$ & $4.0 \times 10^{-10}$ \\
\hline 16p13.11 dup & 0.03 & $0.31(37 / 12029)$ & $0.13(93 / 69289)$ & $2.30(1.57-3.36)$ & $5.7 \times 10^{-5}$ \\
\hline 16p11.2 distal del & 0.0014 & $0.063(13 / 20732)$ & $0.018(5 / 27045)$ & $3.39(1.21-9.52)$ & 0.017 \\
\hline 16p11.2 dup & $3.2 \times 10^{-14}$ & $0.35(58 / 16772)$ & $0.030(19 / 63068)$ & $11.52(6.86-19.34)$ & $2.9 \times 10^{-24}$ \\
\hline 17p12 del & 0.0004 & $0.094(12 / 12773)$ & $0.026(17 / 65402)$ & $3.62(1.73-7.57)$ & 0.0012 \\
\hline $17 q 12$ del & 0.004 & $0.036(5 / 14024)$ & $0.0054(4 / 74447)$ & $6.64(1.78-24.72)$ & 0.0072 \\
\hline 22 q11.2 del & $1.0 \times 10^{-30}$ & $0.29(56 / 19084)$ & 0.00 (0/77 055) & NA $(28.27-\infty)$ & $4.4 \times 10^{-40}$ \\
\hline
\end{tabular}


of patients in this study were recruited on the basis that they have a diagnosis of treatment-resistant schizophrenia according to their psychiatrist and were taking clozapine for that indication. The availability of clinician diagnoses allowed us to exclude the limited number of samples from individuals with diagnoses other than schizophrenia. The convention for psychiatric samples has been that patient inclusion is based on research diagnoses arrived at following detailed interview and phenotyping procedures (as is the case in the CardiffCOGS sample in this study). As genome analysis has become more affordable than the establishment of a formal research diagnosis, the latter has now become the limiting step for exploiting the microarray technology. The CLOZUK sampling method offers a pragmatic approach to recruit unusually large numbers of patients with schizophrenia, and nearly doubles the number of patients with schizophrenia analysed in the previous literature. The use of such samples is supported by evidence that with the use of operationalised criteria, clinician diagnoses of schizophrenia have high specificity and positive predictive values when validated against research-based approaches. $^{30,31}$ Furthermore, we have reported findings that support the validity of the individuals in CLOZUK as a schizophrenia sample with genetic data, by demonstrating that of the most strongly associated schizophrenia alleles in the Psychiatric Genetics Consortium Stage 1, 85\% (66/78) showed the same direction of effect in the CLOZUK sample, sign test $P=1.7 \times 10^{-10} .{ }^{21}$ In the present study, the findings of very similar rates of susceptibility CNVs in the CLOZUK sample compared with previous samples (Table DS6), recruited using conventional methods, support the comparability of the two types of samples.

In order to reduce the potential bias of using different arrays, we used only Illumina platforms and only analysed those probes common to all arrays. We used the $z$-score method to both validate each $\mathrm{CNV}$ and check whether any $\mathrm{CNV}$ in the regions in Table 1 had been missed.

The current study provides support for most previously implicated CNVs, as we found higher rates in the case group than in the control group for 13 of the 15 loci. The support is particularly strong for duplications at $16 \mathrm{p} 11.2\left(P=2.3 \times 10^{-8}\right)$ and at the AS/PWS critical region $(P=0.0055)$ and for deletions at $22 \mathrm{q} 11.2 \quad\left(P=2.2 \times 10^{-6}\right), \quad 1 \mathrm{q} 21.1 \quad(P=0.0027), \quad N R X N 1$ $\left(P=7.7 \times 10^{-4}\right)$ and 15q11.2 $(P=0.046)$. All eight duplications at the AS/PWS region were of maternal origin (online supplement, section 5), thus supporting the original report. ${ }^{6}$ Two loci: deletions at $15 \mathrm{q} 11.2$ and duplications at $16 \mathrm{p} 13.11$, that were not supported in two recent papers, ${ }^{5,10}$ also receive support in the current study and the statistical significance of their overall association with schizophrenia is strengthened by several orders of magnitude (Table 2).

Four of the loci in Table 2 do not surpass a significance threshold that corrects for the multiple testing of large recurrent CNVs $\left(P<4.2 \times 10^{-4}\right)$, or for individual genes $\left(P<2.5 \times 10^{-6}\right)$ (see Method): duplications at VIPR2, and deletions at distal 16p11.2, 17p12 and 17q12.

\section{Burden of schizophrenia-associated CNVs}

Overall, $2.5 \%$ of the case group $v .0 .9 \%$ of the control group carry one or more of these CNVs. This is highly significant in this completely independent data-set $\left(P=1.4 \times 10^{-12}\right)$. They are associated with a range of odds ratios and each locus clearly makes a different contribution to the increase in risk (Table 2). They are also known to increase risk for other disorders, such as epilepsy $\left(15 \mathrm{q} 11.2^{32}\right.$ and $\left.15 \mathrm{q} 13.3^{33}\right)$, congenital heart disease $\left(1 \mathrm{q} 21.1^{34}\right.$ and $\left.22 \mathrm{q} 11.2^{35}\right)$, attention-deficit hyperactivity disorder $\left(16 \mathrm{p} 13.11^{36}\right)$ and obesity (distal $16 \mathrm{p} 11.2^{37}$ ), and all but two (at VIPR2 and 17p12) increase risk for developmental delay and autism spectrum disorders. ${ }^{1}$ The overall contribution is modest but the effect size is sufficiently large to suggest that if seen in a patient, it is very likely to be relevant to the disorder, although not sufficient to account for the disease.

\section{Summary of the findings for the individual loci}

\section{$1 \mathrm{q} 21.1$ deletions and duplications}

Deletions at 1q21.1 were among the first implicated loci. ${ }^{8,38}$ Our new data provide strong support for their role in schizophrenia $(P=0.0027)$, with a frequency in the case group identical to that in previous reports $(0.17 \%),{ }^{14}$ confirming an approximately eightfold excess of deletions among patients, with an extremely strong statistical support of $P=4.1 \times 10^{-13}$ in the combined literature (Table 2). Duplications at this locus have only been implicated in one study, ${ }^{10}$ but with the addition of our data (although not significant on its own), the evidence for duplications at this locus in schizophrenia is now stronger $\left(P=9.9 \times 10^{-5}\right)$.

\section{NRXN1 deletions}

The gene NRXN1 encodes for a presynaptic cell adhesion protein, which binds with postsynaptic proteins called neuroligins and plays a vital role in the formation, maintenance and release of neurotransmitters in synapses. ${ }^{39}$ Exonic deletions disrupting this gene have been consistently implicated in schizophrenia ${ }^{9,10,13,40}$ and autism spectrum disorder. ${ }^{41}$ The current study confirms their role, as we found 11 exonic deletions in the case group $(0.16 \%)$ and none in the control group, $P=7.7 \times 10^{-4}$. This brings the overall significance in the combined literature to $P=1.3 \times 10^{-11}$ (easily surpassing our multiple testing correction threshold for individual genes of $P<2.5 \times 10^{-6}$ ), with approximately a ninefold excess in the case group (Table 2). The positions of exon-disrupting CNVs at this locus in our new data-set are shown in the online supplement, section 4 .

\section{$3 q 29$ deletions}

The role of this CNV in schizophrenia was first reported by Mulle et $a l^{12}$ and confirmed by Levinson et al. ${ }^{10}$ The finding of four individuals in the case group with deletions and none in the control group just fails to reach significance in our independent sample $(P=0.074)$ but can be regarded as supportive independent confirmation. With an overall significance of $P=1.5 \times 10^{-9}$, it is another locus where the evidence for a role in schizophrenia is very strong. Only one such deletion has been found in nearly 70000 controls, indicating it is highly penetrant (Table 2).

\section{WBS duplications}

The reciprocal duplication of the WBS region was first implicated as increasing the risk for autism. ${ }^{42}$ The region was implicated in schizophrenia after the finding of a de novo duplication ${ }^{26}$ and reached statistical support in a large collaborative study. ${ }^{16}$ The finding of three individuals in the case group and one in the control group with this duplication in our study constitutes only modest support, but the overall strength of the evidence remains strong, at $P=6.9 \times 10^{-5}$.

\section{VIPR2 duplications}

The evidence for duplications disrupting this gene comes from two studies that used largely overlapping samples. ${ }^{10,17}$ The overall evidence from the previous literature was modest: $P=0.006 .{ }^{14}$ As we found duplications in six people in the control group and only one in the case group, the evidence in favour of this locus is no longer significant in the combined literature $(P=0.27)$. The 
positions of CNVs at this locus in our new data-set are shown in the online supplement (section 4). Most CNVs at this gene are large and covered with a high number of probes on the arrays (medians of $381 \mathrm{~kb}$ and 66 probes, details in online Table DS4), therefore they should be called reliably on these arrays. We also examined the region with all available probes for CNV calling on the different arrays, and with the $z$-score method, and found no additional CNVs that had been missed. The rate in our control group is slightly higher than in previous studies $(0.095 \% v$. $0.059 \%$, Table 1 and Table DS6), although this difference is not significant $(P=0.4)$.

\section{$15 q 11.2$ deletions}

This was one of the first CNVs implicated in schizophrenia. ${ }^{20,38}$ However, a recent report of a higher rate in controls than that reported in the original paper ${ }^{5}$ and the lack of support in the study by Levinson et $a l^{10}$ clearly indicated the need for replication. Here we found independent significant evidence for association $(P=0.046)$, that strengthens the evidence in the combined analysis to $P=2.5 \times 10^{-10}$ (Table 2).

\section{AS/PWS duplications}

The modest prior evidence for the role of this duplication of the AS/PWS critical region in schizophrenia comes from a single publication with just four observations in patients. ${ }^{6}$ We found another eight CNVs in our case group and none in the control group $(P=0.0055)$, thus substantially strengthening this finding. Even more notably, by using DNA methylation-sensitive highresolution melting-curve analysis of the SNRPN locus, ${ }^{29}$ which does not require DNA from the parents, we found that all eight duplications are of maternal origin, as in the original study. These findings further underline the importance of imprinted genes (those genes subject to parent of origin specific epigenetic regulation) in the aetiology of psychosis and other neurodevelopmental disorders. ${ }^{43}$ These duplications are among the most common genetic susceptibility factors for autism spectrum disorder, where they are found in nearly 1:500 cases. ${ }^{44}$

\section{$15 q 13.3$ deletion}

This is also among the first implicated CNVs in schizophrenia, ${ }^{8,38}$ and received further strong support in the study by Levinson et al..$^{10}$ It was found at a $\sim$ tenfold higher rate in patients with schizophrenia, with a strong statistical support in the previous literature, ${ }^{14} P=2.1 \times 10^{-11}$. Although we found only a twofold excess in our case group in our new sample, at $0.058 \%$ v. $0.032 \%$, $P=0.38$, its role as a susceptibility factor for schizophrenia remains very strong in the combined literature $\left(P=4.0 \times 10^{-10}\right)$. This locus also increases the risk for epilepsy. ${ }^{33,45}$

\section{6p13.11 duplication}

The previous evidence for this CNV comes mostly from a single study ${ }^{7}$ and the overall number of analysed patients with schizophrenia so far, at 5147 individuals, is smaller than in our new data-set. Two recent studies ${ }^{5,10}$ weakened the evidence for this locus, and in the recent review by Malhotra \& Sebat ${ }^{14}$ it had very weak statistical support, $P=0.03$. Here we found $24 \mathrm{CNV}$ s in the case group and 12 in the control group, an excess that just fails to reach significance, $P=0.056$. Combined with the earlier data, our study strengthens the statistical support for this locus in schizophrenia to $P=5.7 \times 10^{-5}$. This $\mathrm{CNV}$ has also been implicated in attention-deficit hyperactivity disorder. ${ }^{36}$

\section{6p11.2 duplication}

This is our strongest finding, with a $P=2.3 \times 10^{-8}$ in the discovery sample alone, and a combined evidence at $P=2.9 \times 10^{-24}$, with an odds ratio of over 11. This duplication is also one of the strongest autism spectrum disorder CNV risk factors. ${ }^{44}$

\section{6p11.2 distal deletion}

This CNV was suggested to confer susceptibility to schizophrenia by Guha et al. ${ }^{18}$ We found no support for this locus in the present study, with two deletions in the control group and none in the case group. However, the lack of deletions among our case group could be as a result of ascertainment bias. Obesity is found in at least $50 \%$ of 16 p 11.2 distal deletion carriers. ${ }^{46}$ Clozapine produces the most severe weight gain among all antipsychotics ${ }^{47}$ and the most common cause for the reluctance of UK psychiatrists to prescribe clozapine is the potential weight gain. ${ }^{48}$ It is therefore possible that psychiatrists are less likely to give clozapine to carriers of 16p11.2 distal deletion (as such patients are more likely to be obese already), thus potentially reducing the frequency of this CNV in the CLOZUK sample. The combined result from the literature retains the statistical support for the role of this locus $(P=0.017)$, but it clearly requires testing in further data-sets.

\section{$17 p 12$ deletion}

This deletion causes the neurological disorder hereditary neuropathy with liability to pressure palsies and was implicated in schizophrenia on the basis of only eight observations in the original case group. ${ }^{20}$ Although the overall statistical evidence is still in favour of it increasing the risk for schizophrenia $(P=0.0012)$, the support is not compelling, raising the need for further replication. It does not increase the risk for either developmental delay or autism, ${ }^{1}$ unlike most other loci discussed here, and it is possible that the original report was a false-positive finding.

\section{$17 q 12$ deletion}

Originally this locus had been known to cause renal cysts and diabetes. It was identified as a susceptibility CNV for schizophrenia with only four observations in the case group ${ }^{19}$ in a study that also implicated its role in autism spectrum disorder. We found only one deletion in a patient, a rate a quarter of that in the original study. ${ }^{19}$ Although we found no deletions in the control group, the overall $P$-value of all data is not sufficiently robust to definitively conclude that this is a schizophrenia susceptibility locus $(P=0.0072)$. Therefore, this locus also requires testing in further schizophrenia data-sets.

\section{2q11.2 deletion}

This was the first CNV implicated in schizophrenia, ${ }^{2,49}$ and has received extensive replication over the years. ${ }^{10}$ It affects $\sim 40$ genes and leads to a variety of physical anomalies. ${ }^{50}$ The latest review found a rate of $0.30 \%$ in patients and $0 \%$ in controls. ${ }^{14}$ This rate is practically identical to our new sample, where we found 20 carriers in the case group (0.29\%) and none in the control group. It remains the most significantly associated $\mathrm{CNV}$ in schizophrenia: $P=4.4 \times 10^{-40}$.

\section{Implications}

Out of 15 previously implicated CNV loci, 11 are now strongly associated with schizophrenia from the combined results of the previous literature and our new data. The evidence for the remaining four loci should be regarded as still equivocal and requiring further investigation. Our findings indicate that approximately $2.5 \%$ of individuals with schizophrenia carry at least one known pathogenic CNV. The odds ratios of these CNVs 
in relation to schizophrenia range between $\sim 2$ and $>50$ and nearly all of them are also associated with a range of other neurodevelopmental disorders, such as autism spectrum disorder and intellectual deficit. ${ }^{1}$ Moreover, a number of the individual pathogenic CNVs are associated with particular physical disease phenotypes such as epilepsy (15q11.2 and 15q13.3), congenital heart disease (1q21.1 and 22q11.2), microcephaly (1q21.1, 3q29 and $16 \mathrm{p} 11.2)$ and obesity (16p11.2 distal). ${ }^{1,32-37,45,51}$ Given their frequency, these findings therefore suggest that routine screening for CNVs should be made available and that the results will have immediate implications for genetic counselling, and given their comorbidity with other medical disorders, for patient management as well. The robust identification of 11 relatively high penetrance risk alleles for schizophrenia also offers promise for biological research aimed at developing animal and cellular models for the identification of novel disease mechanisms and drug targets.

\section{Funding}

The work at Cardiff University was funded by Medical Research Council (MRC) Centre (G0800509) and Program Grants (G0801418) and the European Community's Seventh Framework Programme (HEALTH-F2-2010-241909 (Project EU-GEI), and an MRC PhD Studentship to E.R. M.C.O'D.. M.J.O. and G.K. have received funding from the MRC and Studentship to E.R. M.C.O'D., M.J.O. and G.K. have received funding from the MRC and
the Wellcome Trust, UK. A.R.I. has received funding from the Biotechnology and Biological Sciences Research Council (UK), the Wellcome Trust and the Leverhulme Trust. This work was supported by a clinical research fellowship to J.T.R.W. from the MRC/Welsh Assembly Government and the Margaret Temple Award from the British Medical Association. The schizophrenia samples were genotyped at the Broad Institute, USA, and funded by a philanthropic gift to the Stanley Center for Psychiatric Research.

\section{Acknowledgements}

We thank the participants and clinicians who took part in the Cardiffcogs study. We acknowledge Andrew Iles, David Parslow, Carissa Philipart and Sophie Canton for the work in recruitment, interviewing and rating. For the CLOZUK sample we thank Novartis for their guidance and cooperation. We also thank staff at The Doctor's Laboratory, in particular Lisa Levett and Andrew Levett, for help and advice regarding sample acquisition. We acknowledge Kiran Mantripragada, Lesley Bates, Catherine Bresner and Lucinda We acknowledge Kiran Mantripragada, Lestey
Hopkins for laboratory sample management.

The authors acknowledge the contribution of data from outside sources: (a) Genetic Architecture of Smoking and Smoking Cessation accessed through dbGAP: Study Accession: phs000404.v1.p1. Funding support for genotyping, which was performed at the Center for Inherited Disease Research (CIDR), was provided by 1 X01 HG005274-01 (CIDR is fully funded through a federal contract from the National Institutes of Health to The Johns Hopkins University, contract number HHSN268200782096C). Assistance with genotype cleaning, as well as with general study coordination, was provided by the Gene genotype cleaning, as well as with general study coordination, was provided by the Gene
Environment Association Studies (GENEVA) Coordinating Center (U01 HG004446). Funding
support for collection of data-sets and samples was provided by the Collaborative Genetic Study of Nicotine Dependence (COGEND; P01 CA089392) and the University of Wisconsi Transdisciplinary Tobacco Use Research Center (P50 DA019706, P50 CA084724). (b) High Density SNP Association Analysis of Melanoma: Case-Control and Outcomes Investigation, dbGaP Study Accession: phs000187.v1.p1: research support to collect data and develop an application to support this project was provided by 3P50CA093459, 5P50CA097007, application to support this project was provided by 3P50CA093459, 5P50CA097007, 5R01ES011740, and 5R01CA133996. (c) Genetic Epidemiology of Refractive Error in the
KORA (Kooperative Gesundheitsforschung in der Region Augsburg) Study, dbGaP Study Accession: phs000303.v1.p1. Principal investigators: Dwight Stambolian, University of Pennsylvania, Philadelphia, Pennyslavian, USA; H. Erich Wichmann, Institut für Humangenetik, Helmholtz-Zentrum München, Germany; National Eye Institute, National Institutes of Health, Bethesda, Maryland, USA. Funded by R01 EY020483, National Institutes of Health, Bethesda, Maryland, USA.

Elliott Rees, MRes, James T. R. Walters, PhD, MRCPsych, Lyudmila Georgieva, PhD, Anthony R. Isles, PhD, MRC, Centre for Neuropsychiatric Genetics and $\mathrm{PhD}$, Anthony R. ISles, PhD, MRC, Centre for Neuropsychiatric Genetics and
Genomics, Institute of Psychological Medicine and Clinical Neurosciences, Cardiff Genomics, Institute of Psychological Medicine and Clinical Neurosciences, Cardiff Research, The Broad Institute of MIT and Harvard, Cambridge, Massachuetts, USA; Alexander L. Richards, PhD, Gerwyn Mahoney-Davies, BSC, Sophie E. Legge BSC, Centre for Neuropsychiatric Genetics and Genomics, Institute of Psychological Medicine and Clinical Neurosciences, Cardiff University, Cardiff, UK; Jennifer L. Moran, PhD, Steven A. McCarroll, PhD, Stanley Center for Psychiatric Research, The Broad Institute of MIT and Harvard, Cambridge, Massachuetts, USA; Michael C. O'Donovan, FRCPsych, PhD, Michael J. Owen, FRCPsych, PhD, George Kirov, MRCPsych, PhD, Centre for Neuropsychiatric Genetics and Genomics, Institute of Psychological Medicine and Clinical Neurosciences, Cardiff University, Cardiff, UK

Correspondence: George Kirov, MRC Centre for Neuropsychiatric Genetics and Genomics, Institute of Psychological Medicine and Clinical Neurosciences, Hadyn Ellis Building, Cardiff University, Cardiff CF24 4HQ, UK. Email: kirov@cardiff.ac.uk

First received 22 Apr 2013, final revision 12 Aug 2013, accepted 5 Sep 2013

\section{References}

1 Girirajan S, Rosenfeld JA, Coe BP, Parikh S, Friedman N, Goldstein A, et al Phenotypic heterogeneity of genomic disorders and rare copy-number variants. N Engl J Med 2012; 367: 1321-31.

2 Karayiorgou M, Morris MA, Morrow B, Shprintzen RJ, Goldberg R, Borrow J, et al. Schizophrenia susceptibility associated with interstitial deletions of chromosome 22q11. Proc Natl Acad Sci U S A 1995; 92: 7612-6.

3 Driscoll DA, Salvin J, Sellinger B, Budarf ML, McDonald-McGinn DM, Zackai EH, et al. Prevalence of 22q11 microdeletions in DiGeorge and velocardiofacial syndromes: implications for genetic counselling and prenatal diagnosis. J Med Genet 1993; 30: 813-7.

4 Shprintzen R, Goldberg R, Lewin M, Sidoti E, Berkman M, Argamaso R, et al. A new syndrome involving cleft palate, cardiac anomalies, typical facies, and learning disabilities: velo-cardio-facial syndrome. Cleft Palate J 1978; 15 $56-62$.

5 Grozeva D, Conrad DF, Barnes CP, Hurles M, Owen MJ, O'Donovan MC, et al. Independent estimation of the frequency of rare CNVS in the UK population confirms their role in schizophrenia. Schizophr Res 2012; 135: 1-7.

6 Ingason A, Kirov G, Giegling I, Hansen T, Isles AR, Jakobsen KD, et al. Maternally derived microduplications at 15q11-q13: implication of imprinted genes in psychotic illness. Am J Psychiatry 2011; 168: 408-17.

7 Ingason A, Rujescu D, Cichon S, Sigurdsson E, Sigmundsson T, Pietilainen $\mathrm{OPH}$, et al. Copy number variations of chromosome 16p13.1 region associated with schizophrenia. Mol Psychiatry 2011; 16: 17-25.

8 International Schizophrenia Consortium. Rare chromosomal deletions and duplications increase risk of schizophrenia. Nature 2008; 455: 237-41.

9 Kirov G, Rujescu D, Ingason A, Collier DA, O'Donovan MC, Owen MJ. Neurexin 1 (NRXN1) deletions in schizophrenia. Schizophr Bull 2009; 35 : 851-4.

10 Levinson DF, Duan J, Oh S, Wang K, Sanders AR, Shi J, et al. Copy number variants in schizophrenia: confirmation of five previous findings and new evidence for 3q29 microdeletions and VIPR2 duplications. Am J Psychiatry 2011; 168: 302-16.

11 McCarthy SE, Makarov V, Kirov G, Addington AM, McClellan J, Yoon S, et al. Microduplications of 16p11.2 are associated with schizophrenia. Nat Genet 2009; 41: 1223-7.

12 Mulle JG, Dodd AF, McGrath JA, Wolyniec PS, Mitchell AA, Shetty AC, et al. Microdeletions of 3q29 confer high risk for schizophrenia. Am J Hum Genet 2010; 87: 229-36.

13 Rujescu D, Ingason A, Cichon S, Pietiläinen OP, Barnes MR, Toulopoulou T, et al. Disruption of the neurexin 1 gene is associated with schizophrenia. Hum Mol Genet 2009; 18: 988-96.

14 Malhotra D, Sebat J. CNVs: harbingers of a rare variant revolution in psychiatric genetics. Cell 2012; 148: 1223-41.

15 Kirov G, Rees E, Walters TJ, Escott-Price V, Georgieva L, Richards AL, et al. The penetrance of copy number variations for schizophrenia and developmental delay. Biol Psychiatry 2013; August 27 (Epub ahead of print).

16 Mulle JG, Pulver AE, McGrath JA, Wolyniec PS, Dodd AF, Cutler DJ, et al. Reciprocal duplication of the Williams-Beuren syndrome deletion on chromosome 7q11.23 is associated with schizophrenia. Biol Psychiatry 2013; July 17 (Epub ahead of print).

17 Vacic V, McCarthy S, Malhotra D, Murray F, Chou H-H, Peoples A, et al. Duplications of the neuropeptide receptor gene VIPR2 confer significant risk for schizophrenia. Nature 2011; 471: 499-503.

18 Guha S, Rees E, Darvasi A, Ivanov D, Ikeda M, Bergen SE, et al. Implication of a rare deletion at distal 16p11.2 in schizophrenia. JAMA Psychiatry 2013; 70: 253-60.

19 Moreno-De-Luca D, Mulle JG, Kaminsky EB, Sanders SJ, Myers SM, Adam MP, et al. Deletion $17 \mathrm{q} 12$ is a recurrent copy number variant that confers high risk of autism and schizophrenia. Am J Hum Genet 2010; 87: 618-30.

20 Kirov G, Grozeva D, Norton N, Ivanov D, Mantripragada KK, Holmans P, et al. Support for the involvement of large CNVS in the pathogenesis of schizophrenia. Hum Mol Genet 2009; 18: 1497-503.

21 Hamshere ML, Walters JTR, Smith R, Richards AL, Green E, Grozeva D, et al. Genome-wide significant associations in schizophrenia to ITIH3/4, CACNA1C and SDCCAG8, and extensive replication of associations reported by the Schizophrenia PGC. Mol Psychiatry 2012; 18: 708-12.

22 Wing JK, Babor T, Brugha T, Burke J, Cooper JE, Giel R, et al. SCAN: Schedules for Clinical Assessment in Neuropsychiatry. Arch Gen Psychiatry 1990; 47: 589-93.

23 American Psychiatric Association. Diagnostic and Statistical Manual of Mental Disorders (4th edn) (DSM-IV). APA, 1994. 
24 Wang K, Li M, Hadley D, Liu R, Glessner J, Grant SFA, et al. PennCNV: an integrated hidden Markov model designed for high-resolution copy number variation detection in whole-genome SNP genotyping data. Genome Res 2007; 17: 1665-74

25 Purcell S, Neale B, Todd-Brown K, Thomas L, Ferreira MAR, Bender D, et al. PLINK: a tool set for whole-genome association and population-based linkage analyses. Am J Hum Genet 2007; 81: 559-75.

26 Kirov G, Pocklington AJ, Holmans P, Ivanov D, Ikeda M, Ruderfer D, et al De novo CNV analysis implicates specific abnormalities of postsynaptic signalling complexes in the pathogenesis of schizophrenia. Mol Psychiatry 2012; 17: 142-53.

27 Rees E, Moskvina V, Owen MJ, O'Donovan MC, Kirov G. De novo rates and selection of schizophrenia-associated copy number variants. Biol Psychiatry 2011; 70: 1109-14.

28 Girirajan S, Dennis Megan Y, Baker C, Malig M, Coe Bradley P, Campbell Catarina $D$, et al. Refinement and discovery of new hotspots of copy-number variation associated with autism spectrum disorder. Am J Hum Genet 2013; 92: 221-37.

29 Urraca N, Davis L, Cook EH, Schanen NC, Reiter LT. A single-tube quantitative high-resolution melting curve method for parent-of-origin determination of $15 q$ duplications. Genet Test Mol Biomarkers 2010; 14: 571-6.

30 Ekholm B, Ekholm A, Adolfsson R, Vares M, Ösby U, Sedvall GC, et al. Evaluation of diagnostic procedures in Swedish patients with schizophrenia and related psychoses. Nord J Psychiatry 2005; 59: 457-64.

31 Jakobsen KD, Frederiksen JN, Hansen T, Jansson LB, Parnas J, Werge T. Reliability of clinical ICD-10 schizophrenia diagnoses. Nord J Psychiatry 2005; 59: 209-12.

32 de Kovel CGF, Trucks H, Helbig I, Mefford HC, Baker C, Leu C, et al. Recurren microdeletions at 15q11.2 and 16p13.11 predispose to idiopathic generalized epilepsies. Brain 2010; 133: 23-32.

33 Dibbens LM, Mullen S, Helbig I, Mefford HC, Bayly MA, Bellows S, et al. Familial and sporadic $15 q 13.3$ microdeletions in idiopathic generalized epilepsy: precedent for disorders with complex inheritance. Hum Mol Genet 2009; 18: 3626-31.

34 Christiansen J, Dyck JD, Elyas BG, Lilley M, Bamforth JS, Hicks M, et al. Chromosome 1q21.1 contiguous gene deletion is associated with congenita heart disease. Circ Res 2004; 94: 1429-35.

35 Botto LD, May K, Fernhoff PM, Correa A, Coleman K, Rasmussen SA, et al. A population-based study of the 22q11.2 deletion: phenotype, incidence, and contribution to major birth defects in the population. Pediatrics 2003; 112: 101-7.

36 Williams NM, Zaharieva I, Martin A, Langley K, Mantripragada K, Fossdal R, et al. Rare chromosomal deletions and duplications in attention-deficit hyperactivity disorder: a genome-wide analysis. Lancet 2010; 376: 1401-8.
37 Bochukova EG, Huang N, Keogh J, Henning E, Purmann C, Blaszczyk K, et al. Large, rare chromosomal deletions associated with severe early-onset obesity. Nature 2010; 463: 666-70.

38 Stefansson $\mathrm{H}$, Rujescu D, Cichon S, Pietilainen OPH, Ingason A, Steinberg S, et al. Large recurrent microdeletions associated with schizophrenia. Nature 2008; 455: 232-6.

39 Südhof TC. Neuroligins and neurexins link synaptic function to cognitive disease. Nature 2008; 455: 903-11.

40 Kirov G, Gumus D, Chen W, Norton N, Georgieva L, Sari M, et al. Comparative genome hybridization suggests a role for NRXN1 and APBA2 in schizophrenia. Hum Mol Genet 2008; 17: 458-65.

41 Glessner JT, Wang K, Cai G, Korvatska O, Kim CE, Wood S, et al. Autism genome-wide copy number variation reveals ubiquitin and neuronal genes. Nature 2009; 459: 569-73.

42 Sanders SJ, Ercan-Sencicek AG, Hus V, Luo R, Murtha MT, Moreno-De-Luca D, et al. Multiple recurrent de novo CNVs, including duplications of the 7q11.23 Williams syndrome region, are strongly associated with autism. Neuron 2011; 70: 863-85.

43 Wilkinson LS, Davies W, Isles AR. Genomic imprinting effects on brain development and function. Nat Rev Neurosci 2007; 8: 832-43.

44 Moreno-De-Luca D, Sanders SJ, Willsey AJ, Mulle JG, Lowe JK, Geschwind DH, et al. Using large clinical data sets to infer pathogenicity for rare copy number variants in autism cohorts. Mol Psychiatry 2012; 18: 1090-5.

45 Helbig I, Hartmann C, Mefford HC. The unexpected role of copy number variations in juvenile myoclonic epilepsy. Epilepsy Behav 2013; 28 (suppl 1): S66-8.

46 Bachmann-Gagescu R, Mefford HC, Cowan C, Glew GM, Hing AV, Wallace S, et al. Recurrent 200-kb deletions of 16p11.2 that include the SH2B1 gene are associated with developmental delay and obesity. Genet Med 2010; 12: 641-7.

47 Leadbetter R, Shutty M, Pavalonis D, Vieweg V, Higgins P, Downs M. Clozapine-induced weight gain: prevalence and clinical relevance. Am J Psychiatry 1992; 149: 68-72.

48 Nielsen J, Dahm M, Lublin H, Taylor D. Psychiatrists' attitude towards and knowledge of clozapine treatment. J Psychopharmacol 2010; 24: 965-71.

49 Shprintzen RJ, Goldberg R, Golding-Kushner KJ, Marion RW. Late-onset psychosis in the velo-cardio-facial syndrome. Am J Med Genet 1992; 42 $141-2$.

50 Shprintzen RJ. Velo-cardio-facial syndrome: 30 years of study. Dev Disabil Res Rev 2008; 14: 3-10.

51 Mefford HC, Sharp AJ, Baker C, Itsara A, Jiang Z, Buysse K, et al. Recurrent rearrangements of chromosome 1q21.1 and variable pediatric phenotypes. N Engl J Med 2008; 359: 1685-99. 